\title{
ON THE MAGNETIC-FIELD CONFIGURATION IN SUNSPOTS
}

\author{
O. KJELDSETh MoE* \\ (Institute of Theoretical Astrophysics, \\ University of Oslo, Norway)
}

\begin{abstract}
A BST R A C T
During 1963-67 observations of the magnetic fields in sunspots have been obtained at the Oslo Solar Observatory. For the largest spots the detailed distribution of the magnetic-field strength is found. Based on calculations of line profiles made by the author (Kjeldseth Moe 1967) also the direction of the magnetic field is derived. Observations of the magnetic field of the same spot at several positions on the solar disk give further information regarding the magnetic-field configuration. Our results are in fair agreement with those of Bumba (1962).
\end{abstract}

\section{Introduction}

During the years 1963-67 observations of the magnetic field in sunspots have been obtained at the Oslo Solar Observatory. For our observations we have used the Zeeman triplet $\lambda 5250$ with a Landé factor $g=3$. The entrance slit of the spectrograph is placed at the off axis focus, $f=30 \mathrm{~m}$, of the solar telescope, giving a $27-\mathrm{cm}$ image of the solar disk. The spectrograph has a focal length of $21 \mathrm{~m}$ and is equipped with a blazed Babcock grating with 600 rulings per $\mathrm{mm}$. A more detailed description is given by Brahde (1967). The observations are made in the 5th order. This gives us a linear dispersion of $10 \cdot 1 \mathrm{~mm}$ per Ångström and a resolving power of approximately $1 / 100$ Ångström. In the direction perpendicular to the dispersion $1 \mathrm{~mm}$ in the focus of the spectrograph corresponds to $4.7 \mathrm{sec}$ of arc on the solar disk. The exposure time was less than $15 \mathrm{sec}$.

A quarter-wave analyzer is placed in front of the spectrographic slit. The quarterwave analyzer consists of a grid of mica strips followed by a Glan-Thompson prism. The principal axes of neighbouring mica strips are at right angles to each other, while the axis of the Glan-Thompson prism makes an angle of $45^{\circ}$ to the axes of the mica strips. This arrangement enables us to determine the inclination, $\gamma$, of the magnetic-field vector to the line of sight. The direction of the azimuthal field component has, however, not been measured.

\section{Sources of Error}

Before we discuss the results of the observations we would like to mention some of

* Presented by P. Maltby.

Kiepenheuer (ed.), Structure and Development of Solar Active Regions, 202-210. C I.A.U. 
the sources of error, particularly the instrumental polarization. The light will be polarized in the telescope because of reflections from metal surfaces, in our case from the coelostat- and off-axis mirrors. The amount of polarization in the telescope has been calculated for some typical settings of the coelostat, using data for the refractive index and the absorption coefficient for thin aluminium films.

The greatest contribution to the instrumental polarization comes from the spectrograph, especially from the reflection of light from the grating. The instrumental polarization in the spectrograph has been thoroughly measured by Dr. Maltby and the author for different spectral orders. The effect of instrumental polarization in the spectrograph may, however, be almost completely eliminated by placing a quarterwave analyzer in front of the spectrographic slit. (The very small effect of instrumental polarization in the spectrograph in this case is seen from a comparison of the second and third row in Table 1.)

\section{Table 1}

Inaccuracy in the inclination of the magnetic field to the line of sight, $\Delta \gamma$, due to instrumental polarization and scattered light for inclinations in the neighbourhood of $30^{\circ}$ and $60^{\circ}$, respectively

\begin{tabular}{|c|c|c|}
\hline & & \\
\hline & $\gamma \approx 30^{\circ}$ & $\gamma \approx 60^{\circ}$ \\
\hline Instrumental polarization in the telescope & 5.0 & 13.0 \\
\hline Imperfections in the quarter-wave analyzer & $+2: 0$ & $4: 5$ \\
\hline $\begin{array}{l}\text { Imperfections in the quarter-wave analyzer together with } \\
\text { instrumental polarization in the spectrograph }\end{array}$ & $2: 5$ & $5: 5$ \\
\hline Scattered light $(1 \%)$ from the penumbra & 5.0 & 6.0 \\
\hline
\end{tabular}

The line profile of a Zeeman triplet may be seriously distorted by instrumental polarization. It is, however, possible to find a correct value of the magnetic-field strength when the splitting of the components is complete, even without the use of a quarter-wave analyzer in front of the spectrograph. (For $\lambda 5250$ the components are separated if the field is stronger than 2000 gauss.) The inclination, $\gamma$, of the magneticfield vector to the line of sight may on the other hand only be measured from exposures made with a quarter-wave analyzer in front of the spectrographic slit. But even in this case we must consider the effect of instrumental polarization in the telescope.

Other sources of error, like imperfections in the polarization optics and scattered light, have also been considered. Possible imperfections in the retardation as well as the mounting of the quarter-wave plate may introduce inaccuracies in the observations. It is further possible that the Glan-Thompson prism is not a perfect polarizer. A small amount of light polarized perpendicular to the optical axis of the prism may be transmitted. The inaccuracies in the determination of the angle $\gamma$ are listed in Table 1. 
The values are found from measurements of the quarter-wave analyzer used at the Oslo Solar Observatory.

To correct for scattered light is an extremely tedious task. We have to take into account the different states of polarization of the light scattered from different parts of the spot and from the photosphere. The values in Table 1 are the results of a rough estimate, where we have assumed $1 \%$ scattered light from the penumbra. The magnetic field strength in the umbra and the penumbra was set equal to 2400 and 800 gauss, respectively; while the inclination of the magnetic field to the line of sight was assumed to be the same in the umbra and the penumbra.

The influence of instrumental polarization on the inclination measurements will be greatest for near-transverse fields. This is shown in Table 1, where we have listed the maximum difference, $\Delta \gamma$, between the measured and the real value of the magnetic-field inclination to the line of sight for two values of the inclination: $30^{\circ}$ and $60^{\circ}$. The values in Table 1 are maximum values, and in most cases the difference between the measured and the real inclination will be less than $\Delta \gamma$.

\section{The Strength of the Magnetic Field}

Regarding the results of the magnetic-field strength measurements we will stress in particular these points: We have found the magnetic-field strength to be constant over a greater part of the sunspot umbra. This area of maximum field strength is not necessarily situated at the center of the spot. Further the rate of decrease of the field strength is greatest well outside the umbra/penumbra boundary. It is therefore certainly a difference between the variation of the intensity and the variation of the magnetic-field strength across the spot. The general outline of the isogauss lines seem, however, to follow the umbra/penumbra boundary fairly well. These results are demonstrated in Figures 1 and 2. The only exceptions to these rules are small spots with an umbra diameter less than $5 \mathrm{sec}$ of arc and spots observed under poor observing conditions. In these cases it is likely that the observations will be strongly influenced by scattered light.

In Figures 1 and 2 are shown two examples of isogauss maps prepared for the great spot of March 21 and 22, 1966. The outlines of the umbra and the penumbra are marked with a heavy line. The field strengths on the isogauss lines are given in hundreds of gauss.

In Figure 3 the measured value of $\left(H / H_{\mathrm{Max}}\right)^{2}$ is shown as a function of the distance from the spot center. This curve represents an average of the observations at March 21 and 22 for a number of different position angles in the spot. The magnetic-field strength is constant out to a distance of $0 \cdot 4 r_{u}$ from the spot center ( $r_{u}$ is the umbra radius). Outside this distance the horizontal gradient of the magnetic pressure, $H^{2} / 8 \pi$, may be regarded as nearly constant. The magnetic pressure at the center of the spot is approximately $3.3 \times 10^{5} \mathrm{dyn} / \mathrm{cm}^{2}$ compared with about $10^{5} \mathrm{dyn} / \mathrm{cm}^{2}$ for the gas pressure (if the magnetic field is not force-free). 


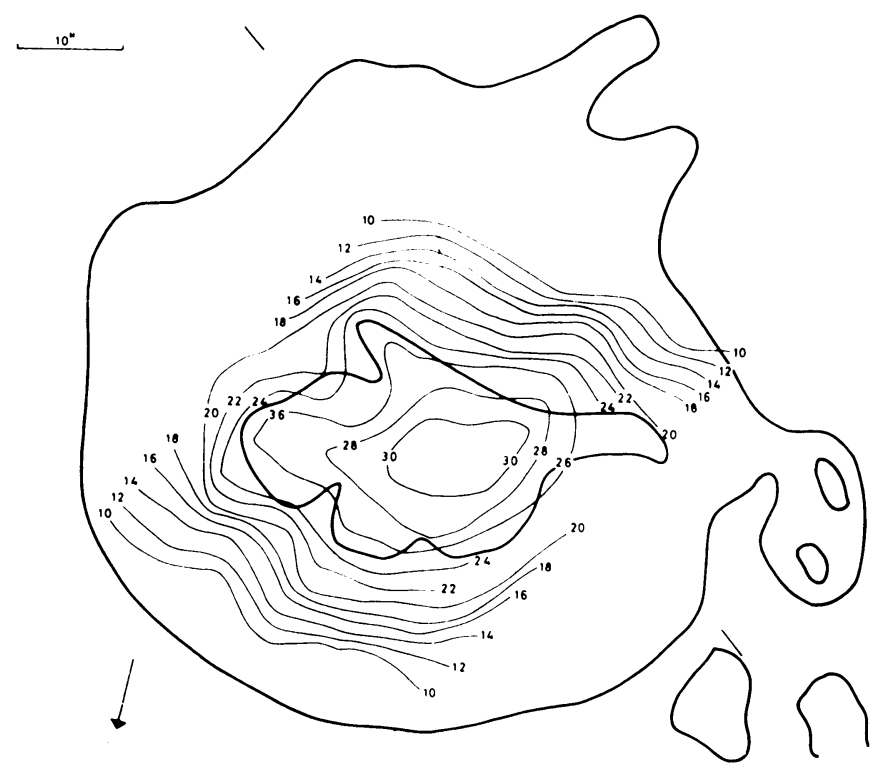

Fig. 1. Isogauss map for the spot of March 21, 1966. The outlines of the umbra and the penumbra are marked with a heavy line. The field strengths on the isogauss lines are given in hundreds of gauss. The arrow points in the direction to the center of the solar disk.

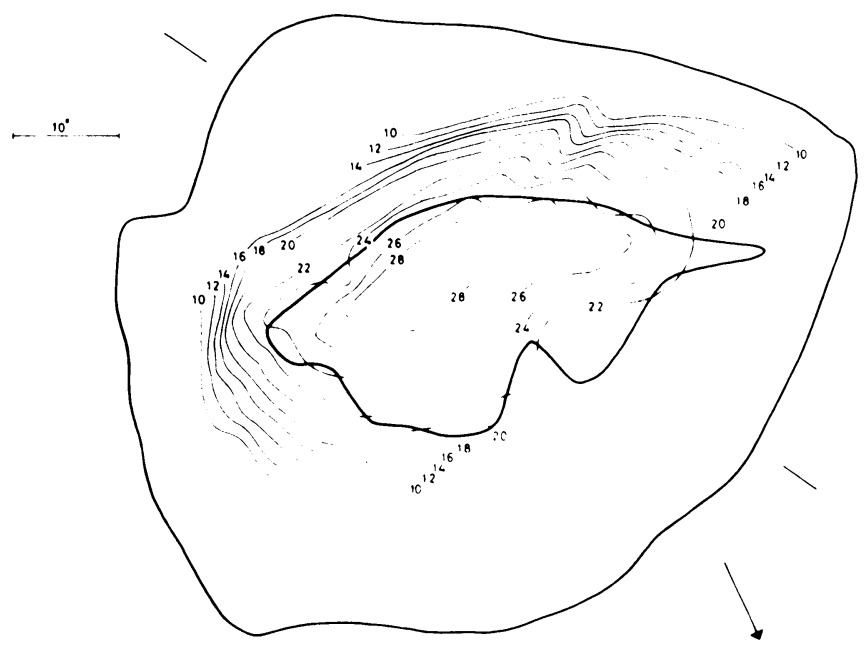

Fig. 2. Isogauss map for the spot of March 22, 1966 (see also text Figure 1). 


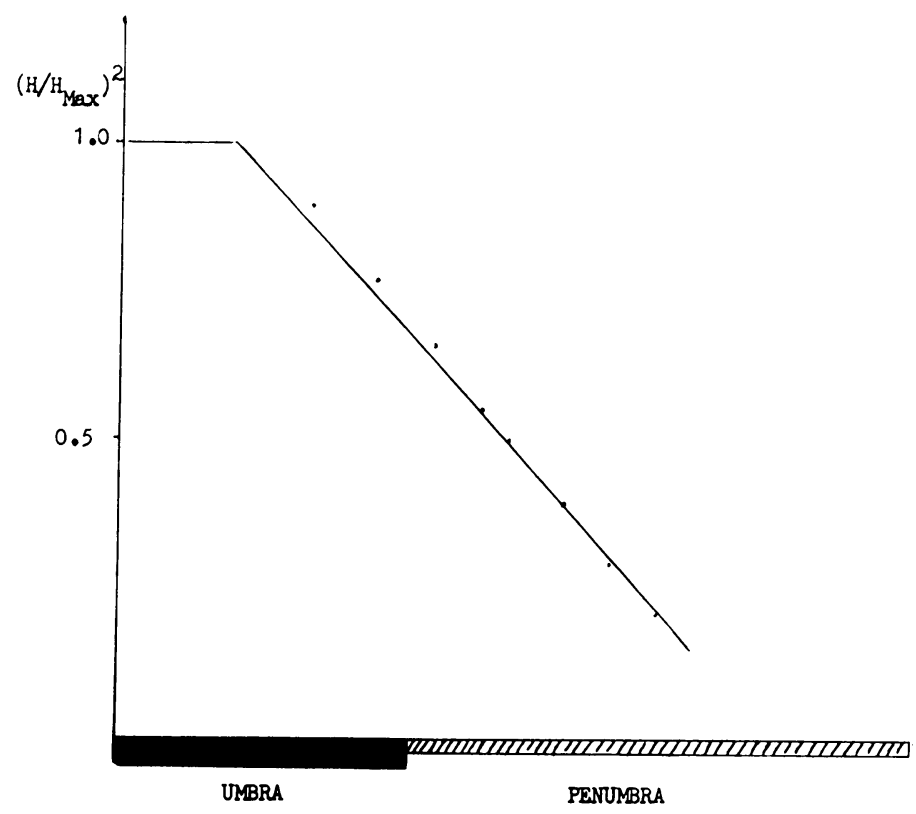

FIG. 3. The measured value of $\left(H / H_{\mathrm{Max}}\right)^{2}$ as function of the position in the spot. The values are the mean of the two days March 21 and 22, 1966, for a number of different position angles in the spot.

\section{The Direction of the Magnetic Field}

The measurements of the inclination of the magnetic-field vector to the line of sight are based on line-profile calculations made by the author (Kjeldseth Moe, 1967). These calculations are performed using a new method for solving Unno's transfer equations for polarized light. Unno (1956) has derived the transfer equations for the Stokes parameters

$$
\begin{aligned}
\frac{\mathrm{d} I}{\mathrm{~d} \tau / \mu} & =\left(1+\eta_{I}\right)(I-B)+\eta_{Q} Q+\eta_{V} V, \\
\frac{\mathrm{d} Q}{\mathrm{~d} \tau / \mu} & =\left(1+\eta_{I}\right) Q+\eta_{Q}(I-B), \\
\frac{\mathrm{d} V}{\mathrm{~d} \tau / \mu} & =\left(1+\eta_{I}\right) V+\eta_{V}(I-B) .
\end{aligned}
$$

Here $I, Q$ and $V$ are the Stokes parameters, $\tau$ is the optical depth in the continuum, $B$ the source function here set equal to the Planck function, and $\mu=\cos \theta$, where $\theta$ is the angle between the solar radius and the beam of light. The quantities $\eta_{I}, \eta_{Q}$ and 
$\eta_{V}$ are defined as:

$$
\begin{aligned}
& \eta_{I}=\frac{1}{\kappa_{0}}\left[\begin{array}{c}
\kappa_{\mathrm{p}} \\
2
\end{array} \sin ^{2} \gamma+\kappa_{\mathrm{l}}+\kappa_{\mathrm{r}}\left(1+\cos ^{2} \gamma\right)\right], \\
& \eta_{Q}=\frac{1}{\kappa_{0}}\left[\frac{\kappa_{\mathrm{p}}}{2}-\frac{\kappa_{\mathrm{l}}+\kappa_{\mathrm{r}}}{4}\right] \sin ^{2} \gamma, \\
& \eta_{V}=\frac{1}{\kappa_{0}} \frac{\kappa_{\mathrm{r}}-\kappa_{\mathrm{l}}}{2} \cos \gamma,
\end{aligned}
$$

where $\kappa_{\mathrm{p}}, \kappa_{1}$ and $\kappa_{\mathrm{r}}$ are the selective absorption coefficients for linearly polarized light and for left-handed and right-handed circularly polarized light, respectively; $\kappa_{0}$ is the continuous absorption coefficient and $\gamma$ is the angle between the magnetic-field vector and the line of sight.

In our solution the variation of $\eta_{0}$ with depth has been taken into account. Here $\eta_{0}$ is the ratio of the line-absorption coefficient in the line center to the continuous absorption coefficient. We find

$$
\begin{aligned}
I= & \begin{array}{l}
Z_{1}+Z_{2} \\
2
\end{array} \\
Q, V & =\underset{\left(\eta_{Q}^{2}+\eta_{V}^{2}\right)^{1 / 2}}{\eta^{1 / 2}} \times \frac{Z_{1}-Z_{2}}{2} .
\end{aligned}
$$

For $Z_{1}$ and $Z_{2}$ we have the expressions

$$
Z_{1,2}=\int_{0}^{\infty} \lambda_{1,2} B \exp \left[-\int_{0}^{\tau} \lambda_{1,2} \mathrm{~d} \tau^{\prime} / \mu\right] \mathrm{d} \tau / \mu,
$$

where $\lambda_{1,2}=1+\eta_{I} \pm\left(\eta_{Q}^{2}+\eta_{V}^{2}\right)^{\frac{1}{2}}$. Equations (3) and (4) are special cases of a more general solution (Kjeldseth Moe, 1967). The magnetic field was supposed to be homogeneous and the contribution to the line formation from scattering was ignored.

Numerical calculations have been made for the Zeeman triplet $\lambda 5250$ using a photospheric model atmosphere. The variation of $\eta_{0}$ with depth was found to be pronounced. Comparing different layers contributing to the line we find that the value of $\eta_{0}$ may change by a factor of the order of $10^{2}$.

From the calculations of the line profile the ratio $r_{\pi} / r_{\sigma_{1}}$ are found as a function of the inclination, $\gamma$, for $\lambda 5250$ in the case of complete splitting $\left(H>2000\right.$ gauss). Here $r_{\pi}$ and $r_{\sigma_{1}}$ are the line contrasts in the $\pi$ - and $\sigma_{1}$-components respectively. The $\sigma_{1}$-component is the strongest of the $\sigma$-components when the line is observed using a quarter-wave analyzer. The result is shown in Figure 4, where Seares' values $r_{\pi} / r_{\sigma_{1}}=\frac{1}{2} \sin ^{2} \gamma / \frac{1}{4}$ $(1+\cos \gamma)^{2}$ have been plotted for comparison. Comparing theoretical and observed values of $r_{\pi} / r_{\sigma_{1}}$ the inclination, $\gamma$, is found. It should, however, be emphasized that the values of $r_{\pi} / r_{\sigma_{1}}$ in Figure 4 are computed for a special model atmosphere (see Kjeldseth Moe, 1967). 


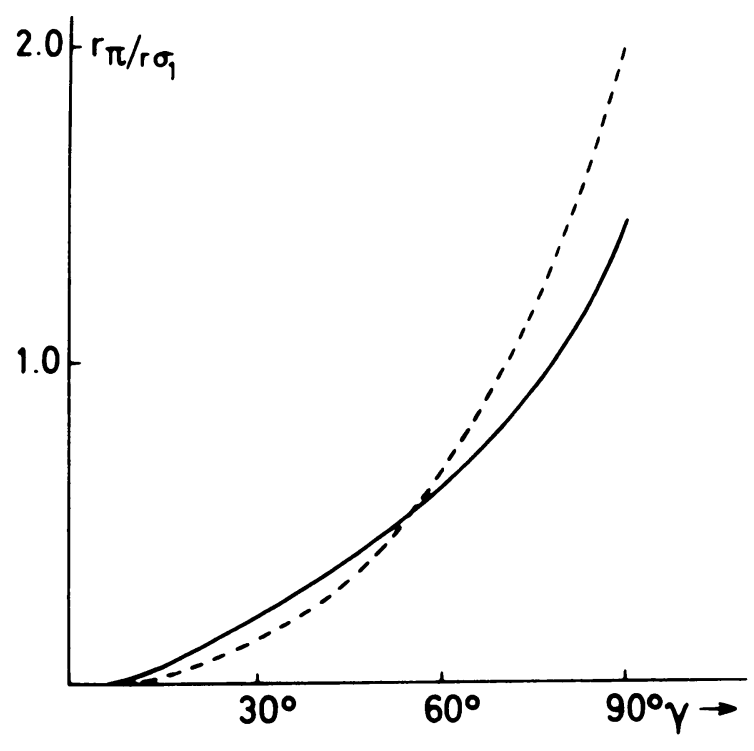

Fig. 4. The ratio between the line depths in the $\pi$-component and the $\sigma_{1}$-component, $r_{\pi} / r_{\sigma_{1}}$, as a function of the inclination, $\gamma$, of the magnetic-field vector to the line of sight. The $\sigma_{1}$-component is the strongest of the two o-components when the line is viewed through a quarter-wave analyzer. For comparison we have plotted the ratio $r_{\pi} / r_{\sigma_{1}}$ computed from Seares' formula (dashed curve).

For high values of $\gamma, \gamma \geqslant 75^{\circ}$, the weakest of the $\sigma$-components, the $\sigma_{2}$-component, will appear. In this case also the ratio $r_{\sigma_{2}} / r_{\sigma_{1}}$ may be used for the measurement of $\gamma$.

The results of the inclination measurements are shown in Table 2 and Figure 5. In Table 2 the inclination, $\gamma$, is listed as a function of the heliocentric angle, $\theta$. The magnetic field is nearly transversal, $\gamma \approx 77^{\circ}$, for all positions of the spot on the solar disk. The variation in $\gamma$ with the heliocentric angle, $\theta$, is very small. This result is in agreement with Bumba's (1962) observations. The values in Table 2 are based on inclination measurements for 10 spots in 30 different positions on the solar disk. In Figure 5 is shown a typical example of the variation in the inclination, $\gamma$, along a section through the spot center. The variations across the spot are within the limits of our accuracy.

\section{Table 2}

The inclination, $\gamma$, of the magnetic field vector to the line of sight as a function of the distance of the spot from the center of the solar disk, $\theta$. Ten spots in thirty different positions on the solar disk have been inspected

$\begin{array}{lccc}\text { Heliographic angle } \theta & 0^{\circ}-35^{\circ} & 35^{\circ}-65^{\circ} & 65^{\circ}-90^{\circ} \\ \text { Inclination } \gamma & 75^{\circ} & 79^{\circ} & 75^{\circ}\end{array}$




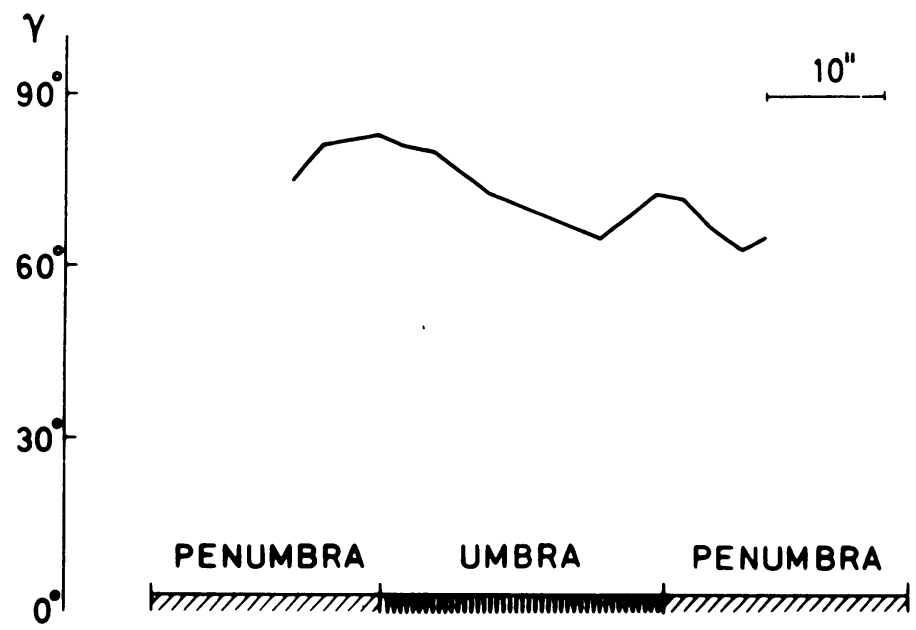

Fig. 5. Variation of the inclination, $\gamma$, along a section through the spot center for the spot of March 22,1966 .

\section{Some Peculiar Spectra}

In Section 4 the relative line contrasts in the $\pi$ - and the $\sigma$-components have been interpreted in terms of the inclination, $\gamma$, of the field lines to the line of sight. This interpretation should be used with caution. Figure 6 is a reproduction of a spectrum taken June 9, 1967, using a quarter-wave analyzer in front of the spectrographic slit. The spot was situated close to the solar limb. During this exposure the spectrographic slit was placed across the central part of the umbra. In the same region there seemed to be a light bridge across the umbra.

In the umbra all the three Zeeman components are clearly visible and the two $\sigma$-components are of about equal strength, corresponding to a magnetic field perpendicular to the line of sight. Also the magnetic field in the umbra on each side of this section through the umbra center was of opposite polarity.

In the penumbra, however, the $\pi$-component is in some places absent while both the $\sigma$-components are present. This effect is observed only in this particular region of the spot and is conspicuous only on 2 of the about 50 spectra taken of the spot that day. This indicates that the effect is not instrumental. The same effect of a lacking $\pi$-component while both the $\sigma$-components are present have also been observed on other occasions, although not very frequently. (The effect is observed in less than 1 spot out of 10.) The reason for this may be that the effect in all cases seems to be limited to a rather small region in the spot. The other spots where the effect was observed were members of very complex groups with many small umbrae inside the same penumbra. 
FIG. 6. A spectrum of $\lambda 5250$ taken June 9, 1967, using a quarter-wave analyzer in front of the spectrographic slit. The spectrographic slit was placed across the central part of the umbra. The spot was situated close to the solar limb.

\section{Acknowledgements}

The author wishes to thank Dr. P. Maltby and Professor E. Jensen for their helpful advice during the investigation.

This research has been sponsored in part by the Air Force Cambridge Research Laboratories through the European Office of Aerospace Research, OAR, United States Air Force, under Contract F61052-67-C-0070.

\section{References}

Brahde, R. (1967) Institute of Theoretical Astrophysics, University of Oslo, Report No. 23. Bumba, V. (1962) B.A.C., 13, 42.

Unno, W. (1956) Publ. astr. Soc. Japan, 8, 108. 